\title{
REDUCTION OF GRAVITY EFFECT ON THE RESULTS OF LOW- FREQUENCY ACCELEROMETER CALIBRATION
}

\author{
G. P. Ripper ${ }^{1}$, C. D. Ferreira ${ }^{2}$, R. S. Dias ${ }^{2}$, G. B. Micheli² \\ ${ }^{1}$ Division of Acoustics and Vibration Metrology - DIAVI, INMETRO., Brazil, gpripper@inmetro.gov.br \\ ${ }^{2}$ Vibration Laboratory - LAVIB, INMETRO, Brazil, rsdias@inmetro.gov.br
}

\begin{abstract}
:
This paper describes a study on the possible sources of systematic errors during the calibration of accelerometers at low-frequencies. This study was carried out on a primary calibration system that uses an air-bearing vibration exciter APS Dynamics 129 and applying the sine-approximation method. Tests performed and actions taken to reduce the effect on experimental results are presented.
\end{abstract}

Keywords: calibration; vibration; lowfrequency; accelerometer.

\section{INTRODUCTION}

The warp of the linear guide can cause a tilt with variable angle of the moving table when it is linearly translated. This might cause a variable effect of local gravity on dc-responsive accelerometers as for instance servo-accelerometers. In the key comparison EURAMET.AUV.V-K3 a larger dispersion among the results of participants was evidenced at the lowest frequencies [1]. For the key comparison CCAUV.V-K3 [2], INMETRO have reported results down to $0.2 \mathrm{~Hz}$. Later on, some efforts were made to extend the lower limit range to $0.1 \mathrm{~Hz}$ but the systematic effect was high compared to the desired uncertainty for the service. This was confirmed during a measurement audit carried out during the peer review of Lavib. In this opportunity (June 2019) we could calibrate a servoaccelerometer from PTB and quantify the problem.

The application of a mathematical correction of the systematic error caused by gravity had been proposed by T. Bruns and S Gazioch in 2016 [3]. We preferred to attack the problem following a different approach. Instead of dealing with the effect, we decided to try to identify and reduce the cause.

Therefore, we searched the responses for the basic questions: Can we clearly identify the cause and consider it is exclusively generated by the warp? Can we minimize the cause of the problem to reduce or even exclude the need of a mathematical correction?
Some experiments were carried out to respond to these questions and the results obtained will be presented in the following sections.

\section{DESCRIPTION OF THE WORK}

The study started with the establishment of a reference condition that was nothing else than the result of sensitivity obtained for a servoaccelerometer Q-Flex QA-3000.

The calibration of accelerometers is usually carried out using two mountings $\left(0^{\circ}\right.$ and $\left.180^{\circ}\right)$. The final sensitivity is the mean of the results obtained at 2 mountings. This basic condition was used as parameter to evaluate the results obtained in our subsequent tests.

\subsection{INITIAL TESTS}

The influence of gravity on the accelerometer was first tested using different orthogonal mounting position $\left(90^{\circ}\right.$ and $\left.270^{\circ}\right)$. Despite of the servoaccelerometer Q-Flex being a based on a cantilever beam design no significant difference was observed. The influence of the distance of the accelerometer from the centre of gravity of the moving table was also tested. A calibration was carried out placing the accelerometer below the mounting plate of the moving table. No significant difference was observed.

The influence of tilt on the measuring points used for calibration was evaluated by using different measuring distances between the laser measuring point and the centre axis of the accelerometer.

Tests after loosening the screws of one end of the guide bar were performed and later after loosening the screws of both ends of the guide bar. Change on the behavior of sensitivity magnitude at low frequencies could be observed at these times.

\subsection{CORRECTION PROPOSED BY PTB}

The correction procedure proposed by PTB have also been tested by INMETRO. Figure 1 presents corrected sensitivity results obtained according to the method proposed by Bruns and Gazioch using 
the same coefficient published by PTB in [3]; using the coefficient determined by INMETRO for its system; original certificate results obtained in 2018 and in 2019; and results with 3 screws loosened at one side the linear guide.

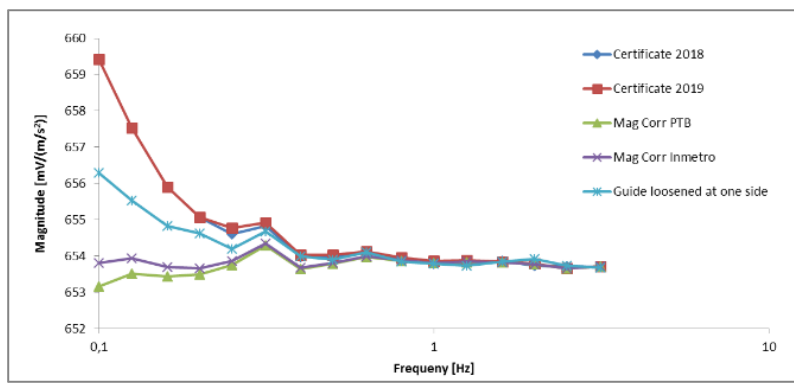

Figure 1: Experimental and corrected results according to method proposed by Bruns and Gazioch.

Figure 1 demonstrates that the repeatability of the results from year to year is good, but the systematic error is too high at $0.1 \mathrm{~Hz}$. The mathematical correction improves the final result obtained but this can be dependent on specific characteristics of the accelerometer under test. The simple test of just loosening the three screws at one side of the linear guide have shown the possibility of achieving an improvement of the sensitivity response measured with the interferometric system by mechanical means.

Therefore, it was decided to quantify the effect caused by this process and take actions in order to improve the straightness of the guide.

\subsection{STRAIGTHNESS MEASUREMENTS}

The procedure applied by INMETRO to measure the straightness comprised the use of an Autocollimator Taylor Robson mounted on an independent seismic block, which is usually used to position a breadboard with the interferometer. The setup is shown in Figure 2.

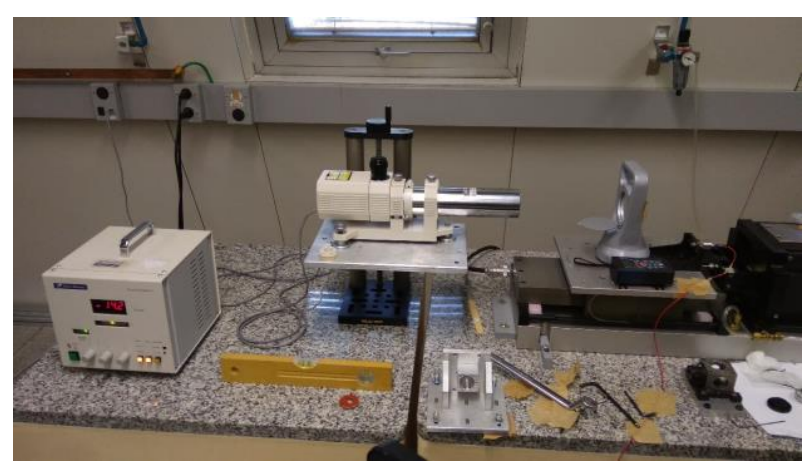

Figure 2: Setup used to measure the straightness of the linear guide of shaker APS 129.

A plane mirror stand was positioned on the top of the moving table and the angle of inclination of this mirror was measured at different positions of the moving table. The zero position was taken as the equilibrium static point with no voltage input to the shaker. This is close to the mid-point of the linear translation guide.

A dc-voltage source was used to provide a voltage of approximately $200 \mathrm{mV}$ to the power amplifier. The gain knob of the amplifier was manually set to place the moving table at different $\mathrm{x}$-positions. The measurement of the $\mathrm{x}$-position was made with a laser distance measurer placed on the top of the table, which was pointed to a fixed reference stand on the same seismic block used to support the autocollimator.

Initially, straightness measurements were made at the mid-point and close to the positive and negative limits of motion used for accelerometer calibrations. Absolute angles measured were -14, 12,6 and $-11,5$ seconds. Normalizing these values in relation to the mid-point angle gives us a better view of the difference of the angle measured at different positions of the linear guide. This is shown in Figure 3.

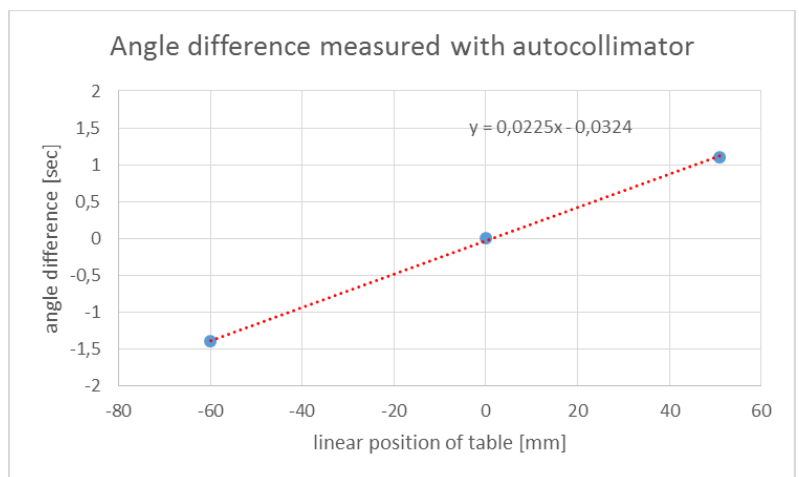

Figure 3: Difference of the angle measured at different positions of the moving table.

New straightness measurements were made while changing the mechanical system as follows:

1. Original measurement conditions

2. Measurements after release of the 3 screws that fix the linear guide to the base

3. Measurements after release of the 6 screws that fix the linear guide to the base

4. Measurements after release of the 6 screws that fix the linear guide to the base and release of the 4 screws that hold the base of the shaker to the seismic block

5. Measurements after re-fixture of the 4 screws that hold the base of the shaker to the seismic block

6. Measurements after re-fixture of the 6 screws that fix the linear guide to the base

After applying the step 6 above, we considered that any stress due to mounting and assembly of the shaker to the seismic block have been released.

A more refined straightness evaluation was made using a positioning resolution of approximately $10 \mathrm{~mm}$. The results obtained are presented in Figure 4. This graph shows that the 
straightness of the system was highly improved. All measurement values were within $\pm 0.2 \mathrm{sec}$ relative to the angle at the reference point.

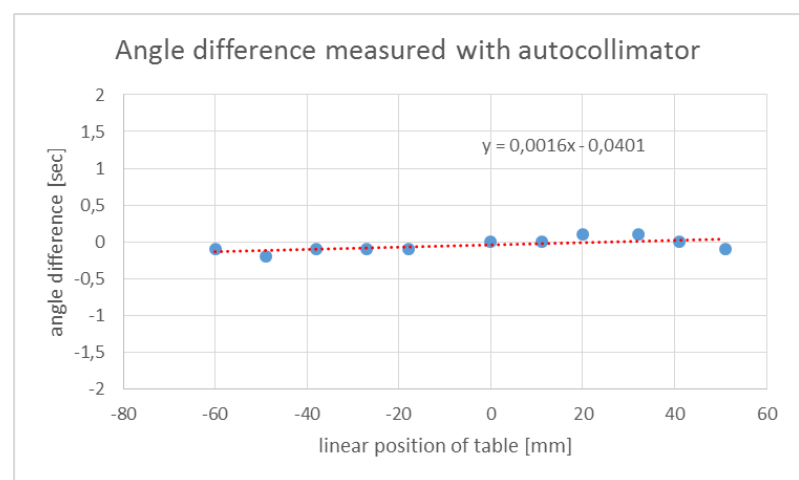

Figure 4: Difference of the angle measured at different positions of the moving table after reassembly of shaker and linear guide.

\subsection{DYNAMIC CALIBRATION OF SERVO- ACCELEROMETER}

Primary calibrations of a high quality dcresponse accelerometer were then carried out to determine the influence of the improvement obtained on the straightness of the linear guide. After the reassembly of the system, with all its screws retightened, calibrations of a servoaccelerometer Allied Signal QA-3000 was calibrated in the frequency range $0.1 \mathrm{~Hz}$ to $80 \mathrm{~Hz}$.

The effect to be considered here is the deviation of the sensitivity magnitude response below $0.4 \mathrm{~Hz}$. The changes are caused by the combination of longer displacements and change of gravity effect due to different angle of the accelerometer while it moves along the linear guide.

The results obtained in the range $0.1 \mathrm{~Hz}$ to $10 \mathrm{~Hz}$ for the accelerometer QA-3000 have shown that the frequency response is now almost flat. Figure 5 presents the magnitude and phase of sensitivity.

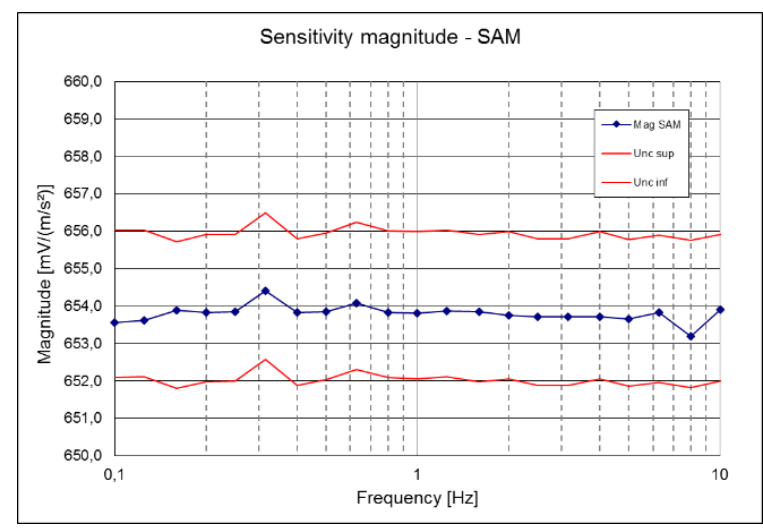

(a) Sensitivity magnitude

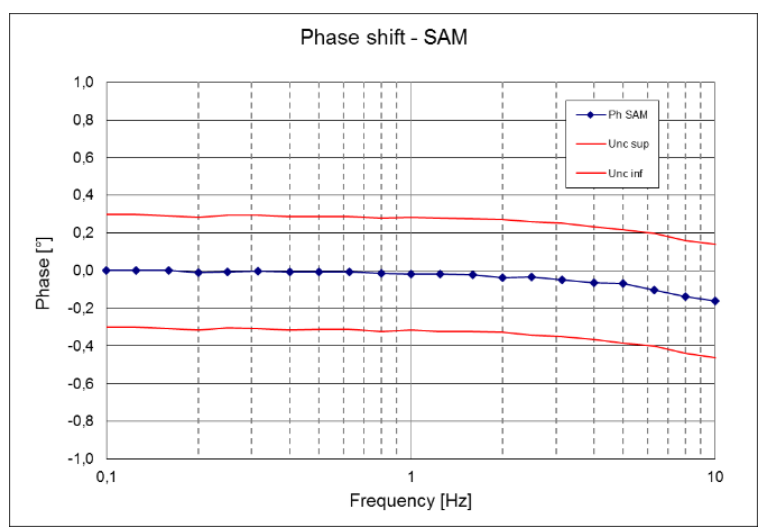

(b) Phase shift

Figure 5: Sensitivity results measured after reassembly of the shaker APS 129

The relative difference of sensitivity magnitude results are now all within $0,1 \%$ taking as reference the value measured at $1 \mathrm{~Hz}$.

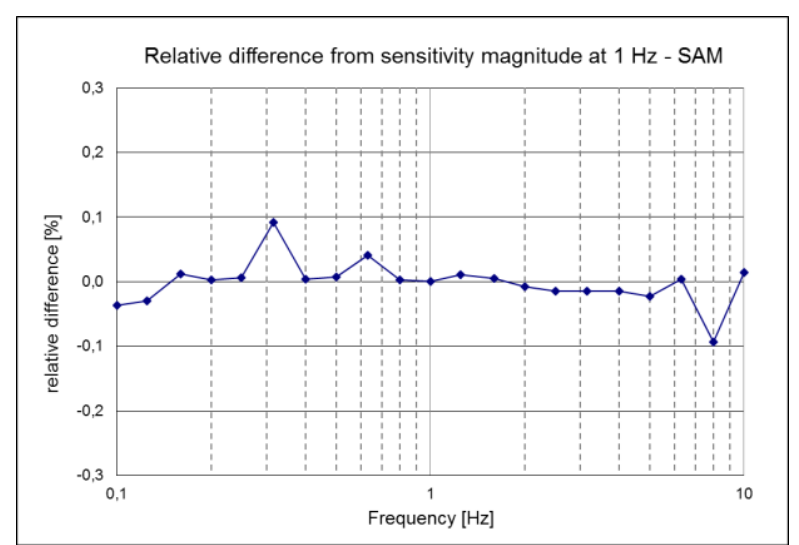

Figure 6: Relative difference of magnitude results relative to the sensitivity value at $1 \mathrm{~Hz}$ - results obtained after reassembly of the shaker APS 129

\subsection{STATIC CALIBRATION OF SERVO- ACCELEROMETER}

As a final check, the calibration of the accelerometer sensitivity at $0 \mathrm{~Hz}$ was carried out by static rotating the accelerometer in the gravity field. The main sensitivity axis of the accelerometer was positioned at different angles relative to the local gravity field and the electrical output was measured. The range of angles from $0^{\circ}$ to $360^{\circ}$ was covered using $20^{\circ}$ steps. Then a sine fit was applied to measured data to obtain the sine amplitude and determine the accelerometer static sensitivity. This was carried out using both a voltmeter Agilent 3458A and the DAQ board NI PCI-6115 used in actual low-frequency accelerometer calibrations applying the sine-approximation method. Due to the difference of input impedance between these two measuring instruments (voltmeter: $10 \mathrm{G} \Omega$ and DAQ $1 \mathrm{M} \Omega$ ), the voltmeter measurement results were corrected by approximately $0.5 \%$ to reflect the same impedance available at the DAQ input channel.

Figure 7 shows that the results obtained statically are in close conformity with the ones obtained 
dynamically, being all within $\pm 0.1 \%$. This demonstrates the improvement obtained in the lowfrequency calibration system.

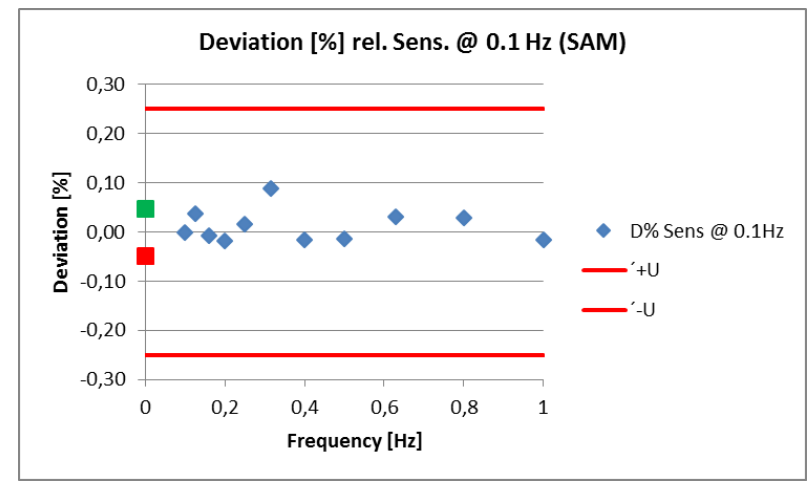

Figure 7: Relative deviation of magnitude results to the sensitivity value at $0.1 \mathrm{~Hz}$ : green square - dc calibration with DAQ, red square - dc calibration with HP3458A (corrected), blue diamond - dynamic calibration results obtained after reassembly of the shaker APS 129 .

\section{SUMMARY}

Small deviations from a purely straight linear motion can cause systematic errors on the calibration of dc-responsive accelerometers at lowfrequencies due to the effect of local gravity. This problem was studied at INMETRO, where differences as large as $0.85 \%$ were observed on primary calibration of accelerometers at $0.1 \mathrm{~Hz}$. The cause identified was a warp of the linear guide, which cause a variable tilting angle of the moving table while it was translated. A procedure was applied to release any possible mechanical stresses that could occur during the mounting of the shaker on the inertial block and try to improve the straightness of the linear guide. This action was successful and allowed us to eliminate the main source of error in calibration below $0.4 \mathrm{~Hz}$. Significant improvement of the calibration results was achieved and eliminated the need of further data correction. The estimated expanded uncertainty of sensitivity is $0.3 \%$ for magnitude and $0.3^{\circ}$ for phase shift in the frequency range from $0.1 \mathrm{~Hz}$ to $10 \mathrm{~Hz}$.

\section{REFERENCES}

[1] Bartoli et al, "Final Report of EURAMET.AUV.VK3", Metrologia 52 09003, 2015.

[2] Sun Qiao et al, "Final report of CCAUV.V-K3: key comparison in the field of acceleration on the complex charge sensitivity", Metrologia 54 09001, 2017.

[3] Th Bruns and S Gazioch, "Correction of shaker flatness deviations in very low frequency primary accelerometer calibration", Metrologia 53, 986, 2016. 